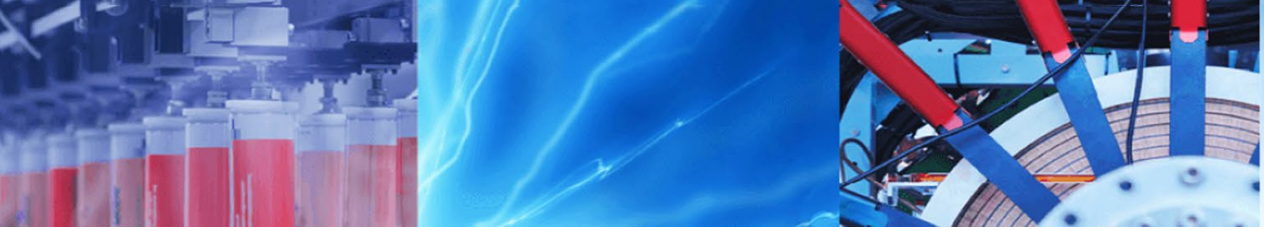

Research Article

\title{
Comparison of artificial neural network and logistic regression model for factors affecting birth weight
}

\author{
Murat Kirişci ${ }^{1}$ (D) \\ () Springer Nature Switzerland AG 2019
}

\begin{abstract}
The aim of this work compares the ANN and logistic regression analysis to determine the factors affecting birth weight. This study included 223 newborn babies. The records of babies born between January 2017 and December 2017 were used. The data were obtained from Beykoz district of Istanbul. ANN and logistic regression analysis of the method obtained based on these records were evaluated. Logistic regression revealed the items GB, MA, GA, NH, BMI, MPPW, MWGP, MsAU, MsCU, MsE as significant factors for BW. The area under the receiver operating characteristic (AuROC) curve 0.941 ( $S D=0.0012$ ) for ANN and 0.909 (SD = 0.019) for Logistic Regression model. The ANNs may be trained with data acquired in various contexts and can consider local expertise, differences, and other variables with uncertain effects on outcome. Although the ANN value is greater than the LR value, these results are very close to each other. This shows us that in terms of their classification ability, these two methods are approximately equal to each other. The results we have seen in our study show that in the medical diagnosis, neither model can change the other. Both models can be used as a complement to help with decision-making. Both models have the potential to help physicians with respect to understanding BW risk factors, risk estimation.
\end{abstract}

Keywords Artificial neural network · Logistic regression $\cdot$ ROC curve $\cdot$ Neonate $\cdot$ Birth weight

\section{Introduction}

Nowadays, Fuzzy sets theory (FST), a useful tool, is used in every field of medicine from public health to surgical systems, from medical imaging to diagnostic systems. Imprecision, uncertainty and vagueness are widespread in medical science. FST has been improved using all values between true and false (or 1 and 0 ), and has become a potential instrument for cope with uncertainty and imprecision. When variables are given linguistically, these variables with FST can be comfortable and easily studied, simplify understandable, and reduce the computational costs. There is also the capability to build systems into human expert experience. FST make this approach a highly exciting option to detailed medical models.

There are a wide variety of NN models which have been advanced which assists physicians in diagnosing the patients more correctly and accurately. NNs make a very generic path of approaching problems. The output of the network represents types of data, which can be divided into groups, that is it consists of categorical variables. Therefore, it is performing prediction. If the output has discrete values, then it is doing classification. Numerous NNs used in disease diagnosis are available in the literature. When studies in the literature are examined, it can be seen that with ANNs, most of the early preterm infants can accurately predict the risk of death.

BW is the initial weight immediately measured when the baby is born. BWs of healthy babies can range from 2500 to $4500 \mathrm{~g}$. An infant with a low birth weight (LBW) may be born prematurely or it may be born smaller than normal. Even, LBW infants can be both small and premature. Some LBW babies may be more at risk for certain health problems. Some of the LBW babies may become

Murat Kirişci, mkirisci@hotmail.com | ${ }^{1}$ Department of Mathematical Education, Hasan Ali Yücel Education Faculty, Istanbul UniversityCerrahpaşa, Vefa, 34470 Fatih, Istanbul, Turkey.

SN Applied Sciences (2019) 1:378 | https://doi.org/10.1007/s42452-019-0391-x 
sick and develop infections in the first days of their lives. In the first days of life, infants who not have these problems may also have long-term problems such as social development and learning disorders. One of the most important factors affecting perinatal morbidity and mortality is the BW of the baby. The birth of the baby on the ideal BW will reduce the perinatal risk. However, BW is a major determinant of infant morbidity and mortality.

Infants born with LBW are more vulnerable to death because they are not immaturity, and this is considered a very important health index [43]. In the literature, the relationship between LBW and infant mortality has been clearly demonstrated. This is the reason why the factors affecting the BW are primarily studied in terms of public health. Many different risk factors have been studied that affect BW. Variables affecting BW are mainly socioeconomic, physical, medical, ethnical and anthropometric categories. Investigating the relationship between one or more of these parameters and the BW will have important consequences. Therefore, it is thought that the factors that may cause babies to be born at LBW and their correction are thought to reduce perinatal morbidity and mortality.

Given the birth outcomes, LBW appears to be a serious problem, especially in developing and undeveloped countries. World Health Organization (WHO) data shows that more than 20 million infants in the world are born LBW, annually. When LBW infant incidence is examined according to the continents, it is seen most in Asia (18.3\%) and least in Europe (6.4\%). According to WHO data, LBW infant incidence is $18.6 \%$ in undeveloped countries, $16.5 \%$ in developing countries and $7.0 \%$ in developed countries.

The main source of data for this study is the live births in Beykoz, Gumussuyu Health Center (BGHS). Due to the "Family Medicine " registration system, BGHS records contain very detailed information about newborn babies and mothers. Using an ANN for input data on neonatal birth weight, we aimed to:

- predict the individual neonatal birth weight from their input data,

- compare the net's performance with that of a logistic regression (LR) model.

\section{Material and method}

\subsection{Type of study}

In this study, factors affecting BW from birth records between January 2017 and December 2017 were examined.

\subsection{Selection of area and samples}

A database was created from the Beykoz District in Istanbul. The records that make up the database consist of the information of the infants followed up by the "Family Medicine System" in BGHS. Records with incomplete information in the dataset have been eliminated.

Beykoz is a relatively rural area in Istanbul. People living in Beykoz usually occur families who have migrated from Anatolia to Istanbul. The lifestyle in Beykoz is mostly rural life rather than urban life. The habits of the people in Beykoz are the cultural lives of the regions where they emigrate. The lives of the people in Beykoz continue according to their economic conditions and lifestyles. Therefore, the life in Beykoz is closer to the disadvantaged zone status.

There are 250.410 people living in the district of Beykoz. The population of the Gümüşsuyu neighborhood is 14.306. Gümüşsuyu is the second most populous neighborhood of the Beykoz district.

It will be useful to examine the impact of the lifestyle of the people living in the rural area on their health. Therefore, the samples of the work consisted of 223 babies born in the Gümüşsuyu neighborhood of Beykoz in Istanbul between January 2017 and December 2017.

\subsection{Types of study variables}

There are 19 independent variables and one dependent dichotomous variable (BW) that form a database. These data were obtained from 223 samples.

The 19 independent variables used in constituting the database are gender of the baby (GB), maternal age (MA), Body Mass Index (BMI), gravida (Gr), parity (Par), nutrition habits $(\mathrm{NH})$, inter-pregnancy interval (IPI), mother's education (MsE), father's education ( $\mathrm{FsE}$ ), antenatal care $(A C)$, gestational age (GA), maternal pre-pregnancy weight (MPPW), maternal weight gain in pregnancy (MWGP), mother's working status (MsWS), income level (IL), mother's alcohol use (MsAU), mother's cigarette use (MsCU), mother's caffeine use (MsCafU), mode of delivery (MD).

From these variables, "antenatal care" has been abolished since each sample cannot be obtained with the correct information. In the same way, this variable has also been eliminated because sufficient and accurate information cannot be obtained about "mother's use of caffeine". In this study, outcome variable is the BW of the baby. Table 1 shows how these variables are selected:

The World Health Organization categorized BMI [44]. It used metric units (kilograms and meters, $\mathrm{kg} / \mathrm{m}^{2}$ ) to calculate the BMls. For BMI $\geq 30$, Obese Class was evaluated as a single class. 


\subsection{Type of analysis}

\subsubsection{Artificial neural networks (ANNs)}

ANNs, one of the research fields of artificial intelligence science, includes studies on learning computers. Today, computers and computer systems have become indispensable parts of our lives. Computers have gained the ability to summarize large amounts of data over time and comment on events using this data, while only accounts or data transfers can be made in the past years. Nowadays, computers are able to make decisions about events and to learn the relationship between them. Problems that cannot be formulated mathematically and cannot be solved can also be solved by computers.

Thanks to their learning features, ANNs are able to provide solutions to the problems that are too complex for traditional techniques. Again, thanks to their learning ability, they can make generalizations in previously unexplored situations using known examples. In ANNs information storage is done with training samples using the training feature provided. With trial and error, the network learns how to do the job on its own. An ANN learns by evaluating the changes in the input set and produces an output to it. The learning process takes place with a learning algorithm that will produce the same output for similar input sets. Training of networks found analogous to nervous structures is similar to the training of a normal livings. The separation of classes from each other is achieved by the stepwise processing of the information

Table 1 Definitions of independent variables

\begin{tabular}{ll}
\hline Variable & Definition (coding) \\
\hline GB & Male (1), Female (2) \\
MA & $19-25(1), \quad 25-35(2), \quad 35+(3)$ \\
GA & $<37(1),>37(2)$ \\
Gr & $1-4$ \\
Par & $1-4$ \\
NH & Regular (1), Irregular (2) \\
IPI (month) & $0-72$ \\
BMI & $<18.5(1), \quad 18.5-24.99, \quad 25.0-29.99(3), \quad>30(4)$ \\
MPPW (kg) & $48-65(1), \quad 65+(2)$ \\
MWGP (kg) & $5-11(1), \quad 11+(2)$ \\
MsAU & Use (1), No use (2) \\
MsCU & Use (1), No use (2) \\
MD & Normal (1), C-section (2) \\
MsE* & P (1), S (2), H (3), U (4) \\
FsE* & P (1), S (2), H (3), U (4) \\
MsWS & Working (1), No working(2) \\
IL (monthly) & $<\$ 1000(1), \quad \$ 1000-\$ 3000(2), \quad \$ 3000+(3)$ \\
\hline
\end{tabular}

*P primary, $S$ secondary, $H$ high, $U$ university taken from the sample set by the learning algorithm. Using ANNs, machines have acquired the abilities such as learning, generalization, classification, estimation and perception.

With the development of computer technology, human beings do almost all of their processing on these innovative technologies and enable new methods to be found. The definition of artificial neurons was first given by McCulloch and Pitts [31]. After these initial definitions, the rapid progress of information technology affected the development of NNs. In the 1980s, the idea that a machine could be thought of as a human being was introduced, and in the 1990s Artificial Neural Networks technology developed rapidly $[21,27,31]$.

NNs are algorithms produced by sampling the shape of the human brain. NNs can collect information about samples, make generalizations, and then decide on those samples using information learned in comparison with samples that they have never seen before. Because of these learning and generalizations, NNs find wide application in many scientific fields and demonstrate their ability to solve complex problems successfully. NNs according to another definition, parallel and distributed information processing structures, which are inspired by the human brain, interconnected by weighted links and each comprising processing elements having their own memory, in other words, computer programs that mimic biological neural networks.

The advantages of using NNs can be expressed as follows:

- Set the rules by giving input and output information during learning.

- Benefit from experience.

- The calculation is cumulative, asynchronous and parallel after learning.

- Memory is allocated and spread out over the network.

- There is error tolerance.

Artificial neural networks are a class of nonlinear regression and discrimination statistical methods. They are of proven value in many areas of medicine. They do not require a priori information regarding the phenomenon, and they make no distributional assumptions. When the appropriate method is used to avoid over fitting (i.e., loss of generalization by fitting the patterns to the test data too precisely), artificial neural networks are usually at least as accurate as classical statistical models, and, depending on the complexity of the phenomena, they can be much more accurate (Fig. 1).

As also shown in Fig. 2, in our model, the number of hidden neurons is 10 . The data were divided into the sets such as for training $70 \%(n=157)$, for validation $15 \%$ 
( $n=33)$ and for testing 15\% $(n=33)$. Levenberg-Marquardt algorithm is chosen for training and Mean Squared Error is chosen for performance.

\subsubsection{Logistic regression (LR)}

Like all regression analyses, the logistic regression (LR) is a predictive analysis. LR is used to describe data and to explain the relationship between one dependent binary variable and one or more nominal, ordinal, interval or ratio-level independent variables.

LR is an algorithm that learns a model for binary classification and is a statistical method for analyzing a dataset in which there are one or more independent variables that determine an outcome.

LR models generally include only the variables that are considered "important" in predicting an outcome. With use of $p$ values, the importance of variables is defined in terms of the statistical significance of the coefficients for the variables. The significance criterion $p \leq .05$ is commonly used when testing for the statistical significance of variables; however, such criteria can vary depending on the amount of available data. For example, if the number of observations is very large, predictors with small effects on the outcome can also become significant. To avoid exaggerating

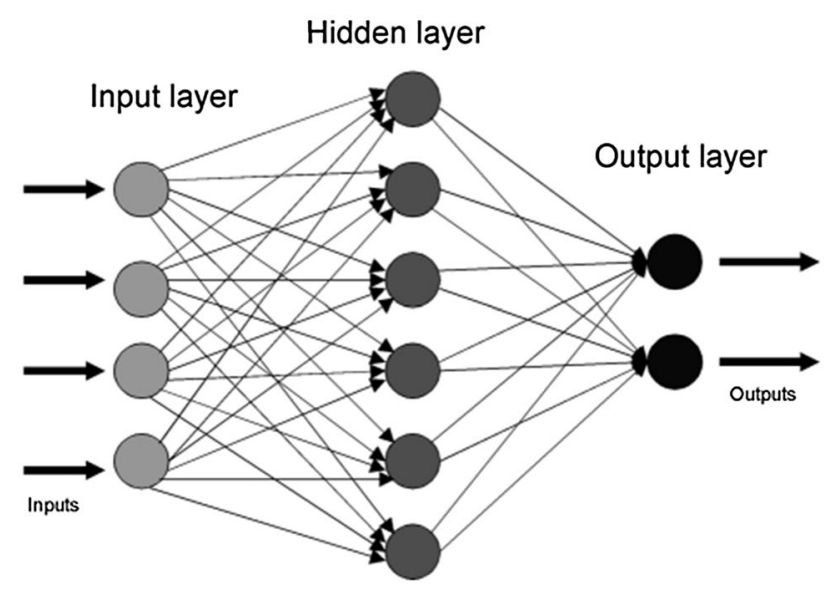

Fig. 1 Neural network model layers: input, hidden, output. Source: Ramesh et al. [35] the significance of these predictors, a more stringent criterion (e.g., $p \leq .001$ ) can be used.

The name of the $L R$ analysis is derived from the logit transformation applied to the dependent variable. If the dependent variable is a binary categorical variable (or multivariate), this analysis is called binary (multinomial) logistic regression analysis.

Mathematically, LR is based on probability, odds and logarithm of odds. In LR, odds is defined as divide of the probability of have an event and the probability of not have an event. That is, for $f(E)$ is a probability of an event $E$ and $1-f(E)$ is a probability of no an event,

odds $=\frac{f(E)}{1-f(E)^{\prime}}$.

The concept of Logit is defined natural logarithm of odds ratio.

In LR, $Y$ is the likelihood that a result or the other will occur, based on a nonlinear model that results in the best possible linear combination of the predictor variables. Then,

$Y_{i}=\frac{e^{T}}{1-e^{T}}$

where $Y$ is the estimated probability that the ith item belongs to one of the categories of the dependent variable and $T$ is defined as

$T=B_{0}+B_{1} X_{1}+\cdots+B_{k} X_{k}$.

Then,

$\ln \left(\frac{Y}{1-Y}\right)=B_{0}+B_{1} X_{1}+\cdots+B_{k} X_{k}$.

In LR, the probability values range from 0 to 1 . However, in order for the predicted values not to fall outside this range, the possibility is rephrasing as odds and the logit value is calculated.

\subsection{ANN and LR in medicine}

Because of the significantly flexibility of input data, ANNs have proven helpful in medicine. ANNs have various applications in medical science $[6,8,24,26,30]$.
Fig. 2 Our neural network structure

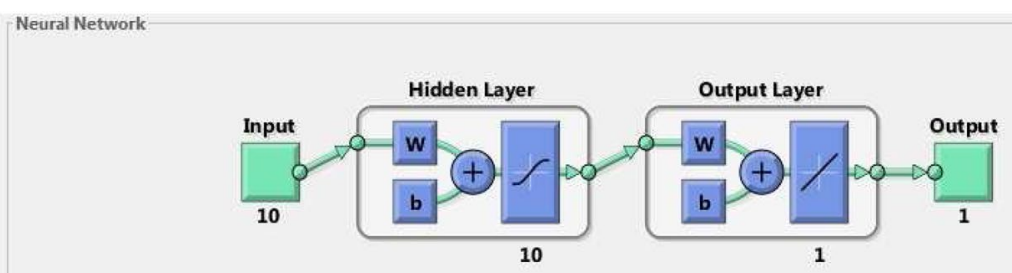


Itchhaporiaa et al. [24] characterizes the computer technology of ANNs and investigates the usage of ANN technology in clinical cardiovascular research. Amato et al. [2] study and discuss the philosophy, capabilities, and limitations of ANNs in medical diagnosis. In this discuss, medical diagnosis examples such as cardiovascular diseases, cancer, diabetes are presented. The method of classifying cancers to specific diagnostic categories based on their gene was developed using ANNs [26]. Wang et. al. [41] improved an ANN model. The model can be used to fast detect those at high risk of Type 2 Diabetes Mellitus, and monitor undiagnosed Type 2 Diabetes Mellitus patient in rural adults using demographic, lifestyle, and anthropometric data.

Firstly, the authors describe the fundamentals of NNs and then discuss the main application fields of NN technology in medicine, in [32]. Baxt [8] discussed the applications of NN in medicine and gave some examples such as myocardial infarction, analysis of plain radiographs of focal bone lesions, waveform analysis. Multilayer NN, probabilistic $\mathrm{NN}$ and learning vector quantization $\mathrm{NN}$ results were obtained for thyroid disease diagnosis and these results were compared. It is seen that the results obtained are better than the results presented in previous studies [37].

A geometrical interpretation of fuzzy sets using points in a fuzzy hypercube is given [28]. Using this geometric interpretation, fuzzy logic applications in medicine and bioinformatics are explained [40]. Er et al. [18] used the ANNs for diagnosis of tuberculosis. Dey et al. [16] used an ANN software program located on the market to differentiate between prognostically good and bad chronic myeloid leukemia cases. An AAN model is built for the detection of carcinoma in effusion cytology [5]. Devi et al. [15] discussed NNs architectures related to the cervical cancer for detection methods at the earliest stage.

A new intelligent method in the clinical diagnosis of thoracic lung cancer surgery is proposed, that helps doctors in patient selection and identifies the risk of death in patients after surgery [23]. One of the causes of adverse neonatal outcomes is maternal obesity. Therefore, at the end of pregnancy, ANN models based on maternal weight status and clinical data have been developed to estimate reliable maternal blood concentrations of oxidative stress and adipokines biomarkers [36].

Austin and Merlo [3] described group of analysis. In this group, the fitting of multilevel logistic regression models are complemented.

About the fit of logistic regression models, a conspectus of several easily operated methods offered by Hosmer et al. [22]. Logistic regression is introduced by Wiest et al. [42] and exemplified how logistic regression works with data from nasal CPAP (continuous positive airway pressure) or Intubation at Birth Trial (COIN).
The optimal selection of patients eligible for pegfilgrastim prophylaxis is crucial. Because this choice is vital for medical economy. Using multivariate ordered logistic regression analysis, the significant factors for eligible patients to maintain the relative dose intensity (RDI) are found as younger age, anemia (less), and administration of pegfilgrastim 24-72 $\mathrm{h}$ after chemotherapy [25].

\subsection{Comparison of analysis}

Analysis methods of this study are ANN and LR. After data were checked, verified, edited, data entered into MATLAB 2015b for ANN and IBM SPSS Statistics 21 for LR.

The ANN and the LR gave the BW of each neonate. For both analysis methods, the predicted results and actual results were evaluated for performance. The ROC (receiver operating characteristic) curve was used when evaluating the performances of the ANN and LR methods (Fig. 3). Sensitivity analysis was performed at various specificity levels for the prediction accuracy of these methods and the analysis results were compared (Table 4).

When recently published articles are examined, it seems that the work done with LR models has increased. In addition, the frequency of LR models and ANNs comparison studies is also striking.

Breast cancer risk based on mammographic descriptors and demographic risk factors is estimated by LR and ANN and discussed advantages and disadvantages of these models [4].

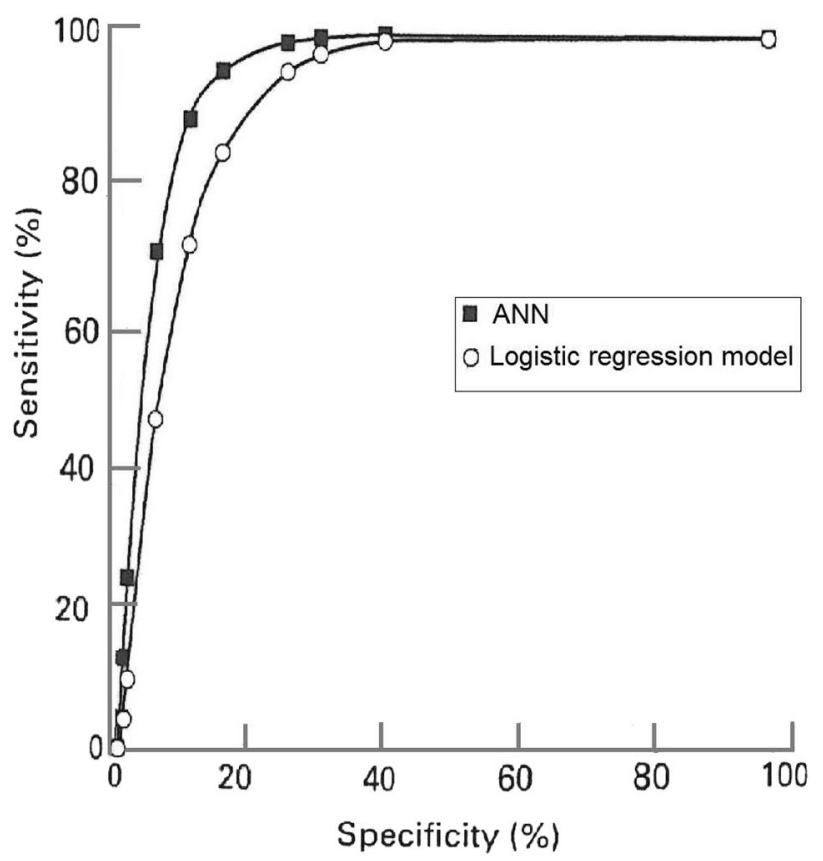

Fig. 3 Comparison of ANN and logistic regression (AuROC) 
In almost all medical branches, studies comparing ANN and LR results are available. Cazzaniga et al. [11] used the ANN and LR model for cirrhosis in chronic hepatitis C patients. As a result of this comparison, they showed that the ANNs were slightly more exact than the LR.

Cucchetti et al. [14] presented a paper related to the ability of the ANN method which is predict tumor grade based on preoperative clinical and radiological variables. In this paper, the performance of the ANN and the performance of the LR model are compared.

Hon-Yi et al. [20] used the ANN for predicting in-hospital survival in (HCC) surgery patients in Taiwan. Further, they compared the predictive accuracy of ANN with that of LR model.

\section{Results}

One of the contributing factors to the United Nations Millennium Development Goals is to reduce the low birth weight and child mortality of babies born. In this study, various factors affecting birth weight were examined. Beykoz, a rural area, was chosen for the study.

The comparison of prediction models in this study showed that accuracy in predicting BW was significantly higher in the ANN model than in the LR model. The prediction model in this study was constructed using registry data from the BGHS. Compared to data obtained by singlecenter series studies, data from registry studies provide a better overview of practices in large populations while avoiding referral bias or bias reflecting the practices of individual surgeons or institutions.

Because ANNs use a dynamic approach to analyzing BW, they can modify their internal structure in relation to a functional objective by bottom-up computation (i.e., by using the data themselves to generate the model). ANNs can simultaneously handle numerous variables by building models with reference to outliers and nonlinear interactions among variables. Whereas conventional statistical methods reveal parameters that are significant only for the overall population, ANNs include parameters that are significant at the individual level even if they are not

Table 2 Averages

\begin{tabular}{ll}
\hline Variable & Average \\
\hline Birth weight $(\mathrm{g})$ & 3130.45 \\
Maternal age & 29.74 \\
Gestational age (week) & 38.2 \\
$\mathrm{BMI}\left(\mathrm{kg} / \mathrm{m}^{2}\right)$ & 25.3 \\
Maternal pre-pregnancy weight $(\mathrm{kg})$ & 66 \\
Maternal weight gain in pregnancy & 13.7 \\
\hline
\end{tabular}

SN Applied Sciences significant for the overall population. Unlike other standard statistical tests, ANNs can also manage complexity even when the sample size is small and even when the ratio between variables and records is unbalanced. That is, ANNs avoid the dimensionality problem.

The averages are given in Table 2 . The mean of mothers who are first birth $39.46 \%$.

Logistic regression analysis was undertaken and all variables that had a significant relationship with low birth weight were entered into the model.

Generally, it is known that female babies average born between 60 and $120 \mathrm{~g}$ less than male babies. Similarly, we found such a relationship between the birth weight of male infants and the birth weight of female infants in this study.

The effects of maternal age on BW was assessed. Participants are between 19 and 43 years old. If the maternal age is advanced during birth, BWs of babies are also heavier. If the mother is under 20 years of age, there is a possibility that the birth weight of the baby is also low. There was a significant progression of birth weight with advancing age. In this study, it was found that the LBW risk was high in the under 20-year-old mothers. Maternal age was positively associated with weight, BMI, as expected, and negatively associated with gestational weight gain.

Table 1 presents the variables and coding of these variables. An LR model was established using these variables. $\mathrm{LR}$ revealed the items GB, MA, GA, NH, BMI, MPPW, MWGP, $\mathrm{MsAU}, \mathrm{MsCU}, \mathrm{MsE}$ as significant factors for BW (Table 3). Other factors did not reach significance when the odds ratio was calculated (that is, $p$ value is non-significant).

Information on the gestational age of the newborn derived from vital statistics is used extensively for surveillance of preterm birth, to determine optimum GA for delivery, and to advance understanding of the etiologies of adverse perinatal outcome. In this work, infants whose BW is less than $2500 \mathrm{~g}$ are $13.89 \%$ (95\% Cl:12.05-14.33). These

Table 3 Logistic regression model for ten predictor variables

\begin{tabular}{llll}
\hline & OR & $95 \% \mathrm{Cl}$ & $p$ value \\
\hline GB & 5.28 & $4.80-6.97$ & 0.0001 \\
MA & 1.04 & $0.86-1.09$ & 0.002 \\
GA & 9.12 & $8.79-10.98$ & 0.0001 \\
NH & 1.10 & $0.94-2.75$ & 0.0026 \\
BMI & 1.62 & $0.97-2.75$ & 0.003 \\
MPPW & 2.51 & $1.87-3.89$ & 0.0001 \\
MWGP & 1.41 & $0.82-2.23$ & 0.02 \\
MsAU & 8.86 & $7.23-10.54$ & 0.0001 \\
MsCU & 9.18 & $8.42-11.07$ & 0.0001 \\
MsE & 1.35 & $0.74-2.48$ & 0.038 \\
\hline
\end{tabular}

OR odds ratio, $\mathrm{Cl}$ confidence interval 
babies were born between 34 and 37 weeks. This data set showed that there was a close relationship between gestational age and birth weight.

Malnutrition during pregnancy is one of the most important causes of LBW. NH can also vary according to cultures. The IL of people in the selected region for this study is not low. However, the rural life style of the region has an important impact on nutrition. Although nutritional resources are more organic than urban life, irregular and unhealthy nutrition is very common. From the analysis on the dataset of this study, it was seen that the irregular $\mathrm{NH}$ was the effect on the BW. It was found that $22.2 \%$ of the mothers were fed irregularly before and during pregnancy. When irregularly fed women gave birth, the infants were found to have $41.6 \%$ LBW, $33.3 \%$ (OW) and $25.1 \%$ normal weight (NW).

Pre-pregnancy health status and BMI affect weight gain during pregnancy. The study was carried out according to the BP values categorized by the World Health Organization [44]. It used metric units (kilograms and meters) to calculate the BMIs. For BMI $\geq 30$, Obese Class was evaluated as a single class. As can be seen from Table 3, there is a significant relationship between $\mathrm{BMI}$ and $\mathrm{BW}$.

If the weight is normal before pregnancy, it is recommended to gain weight between 11 and $15 \mathrm{~kg}$ during pregnancy [45]. The subject of MWGP and its effect on $\mathrm{BW}$ is of tremendous interest. Most studies on this subject since the 70's have focused on poor MWGP and its

Table 4 ANN inputs and AuROC curves of ANNs contribution to producing LBW infants. Once this became well established, a trend toward recommendation of increased MWGP began. This was in sharp contrast to earlier recommendations, which had emphasized restriction of excessive weight gain [12]. In our model, MWGP as data is closely related to BW. Less than half of women had adequate gestational weight gain.

Smoking is the second most common cause of death in the world. Increasing cigarette consumption in the female population in a society also ascends the risk of pregnancy related to cigarette smoking $[1,10,13,19,29,38]$. The most consistent finding associated with maternal smoking during pregnancy is LBW. Most studies find a difference of 200-250 $\mathrm{g}$ between babies of mothers who smoke and those who do not. In the data set examined, among participants, $9.6 \%$ of women aged $19-25$ years and $7.9 \%$ of women over 25 years of age are smoking while pregnant. It has been observed that cigarette smoking has a significant effect on the BW in pregnancy.

Alcohol use can result in harm not only to the drinker, but also to other individuals associated with the drinker. Alcohol use during pregnancy has been established as a risk factor for adverse pregnancy outcomes including stillbirth, spontaneous abortion, premature birth, intrauterine growth retardation, and LBW. Mothers who use alcohol in pregnancy are $12 \%$, in this study. $69 \%$ of the mothers who used alcohol were found irregular $\mathrm{NH}$. As expected, there was a relationship between alcohol consumption and BW in the model.

Preterm or LBW are often encountered in women considered to be of lower socioeconomic status $[7,9,17,33$, $34,39]$. In our study, maternal education level and LBW relation was determined as $4.63 \%$. Further, maternal education was positively associated with weight, and BMI was positively associated with weight.

Socioeconomic disadvantage does not cause low birth weight directly. However, socioeconomic disadvantage can limit access to medical care, restrict nutrition during pregnancy, produce stress, and result in behaviors and maladaptive coping strategies such as teenager pregnancy, smoking, and drug use that do exert direct biological affects on BW. According to the model established in this study, there was no significant relationship between BW and variables of gravida, parity, inter-pregnancy interval, mode of delivery, father's education and mother's working status, income level. For example, it is known that people in the region where we work do not have a very bad income level. However, the effects of the rural lifestyle and the consumption habits influence the birth weight. In other words, the income level is not effective if the babies born in this region are LBW.

The resulting areas under the ROC curves reflecting each ANN's performances are given in Table 4. Table 5 shows that 
Table 5 Comparison of prediction accuracy of ANN and LR

\begin{tabular}{|c|c|c|c|c|}
\hline & \multicolumn{4}{|l|}{ Specificity (\%) } \\
\hline & 80 & 85 & 90 & 95 \\
\hline $\begin{array}{l}\text { ANN Sensitivity } \\
\text { (\%) With (95\% } \\
\text { Cl) }\end{array}$ & $96.4(88.1-97.6)$ & $93.9(84.1-98.2)$ & $88.4(76.3-92.6)$ & $70.2(55.8-85.9)$ \\
\hline $\begin{array}{l}\text { LR Sensitivity } \\
\text { (\%) With (95\% } \\
\text { CI) }\end{array}$ & $89.8(80.2-93.9)$ & $83.4(70.5-91.7)$ & $72.6(57.4-83.2)$ & $50.7(33.1-69.0)$ \\
\hline$p$ value & 0.033 & 0.014 & 0.004 & 0.001 \\
\hline
\end{tabular}

$\mathrm{Cl}$ confidence interval

when adjusted for a specificity level of $80,85,90$, or $95 \%$, in terms of sensitivity, the predictions of the ANN were significantly better than the logistic regression model.

As can be seen from Fig. 3, the area under the receiver operating characteristic (AuROC) curve 0.941 $(S D=0.0012)$ for $A N N$ and $0.909(S D=0.019)$ for $L R$ model. That is, the performance of ANN seems to be significantly better than the performance of $L R$.

The ANN approach is apparently superior to linear regression for describing systems. The ANNs may be trained with data acquired in various contexts and can consider local expertise, differences, and other variables with uncertain effects on outcome.

\section{Conclusion}

The aim of this work was to compare the prediction classification efficiency of ANN and LR in predicting BW. It has been seen that ANN gives more accurate results than $L R$ when estimating BW.

If choose $p$ value as significance at $5 \%$ statistical level, values below $5 \%$ are significant and those with high coefficient absolute values influence more the outcome of interest.

Depending on the purpose of the analysis to be performed or the characteristics of the data collected for this purpose, the model to be selected may be more appropriate than another model. The main purpose of model development is to examine the causal relationship between independent and dependent variables. In such a study, the first option is usually the LR. If the main purpose is outcome classification and considerable mutually effects or complicated nonlinearities exist in the data set, then using the ANN can be especially beneficial. In addition, it requires less adjective statistical training and can be improved using multiple different training algorithms. A constraint of NN models is that standardized coefficients and odds ratios corresponding to each variable cannot be easily computed and offered as they are in regression models.

ANN is very powerful as a method. That is, ANN does not need a high level of operator decision. Therefore, it uses a sophisticated nonlinear model to attain high classification performance. Moreover, logistic regression may constitute many sets of models that yield similar performances, and the operator will need to make intellectual decisions to select the best models.

In our work, both ANN and LR performed well. However, when compared numerically, it appears that the performance of the ANN is higher than that of the LR model (ANN 0.941, LR 0.909). That is, although the ANN value is greater than the $L R$ value, these results are very close to each other. This shows us that in terms of their classification ability, these two methods are approximately equal to each other.

The results we have seen in our study show that in the medical diagnosis, neither model can change the other. Both models can be used as a complement to help with decision-making. Both models have the potential to help physicians with respect to understanding BW risk factors, risk estimation.

Data availability The data used to support the findings of this study are available in the author. The author is ready to share on request.

\section{Compliance with ethical standards}

Conflict of interest The author(s) declare that they have no competing interests.

\section{References}

1. Agrawal A, Scherrer JF, Grant JD, Sartor C, Pergaida ML, Duncan $A E$ et al (2010) The effects of maternal smoking during preg$J$ (2013) Artificial neural networks in medical diagnosis. J Appl Med 11(2):47-58

3. Austin PC, Merlo J (2017) Intermediate and advanced topics in multilevel logistic regression analysis. Stat Med 36:3257-3277

4. Ayer T, Chhatwal J, Alagoz O, Kahn CE, Woods RW, Burnside ES (2010) Comparison of logistic regression and artificial neural network models in breast cancer risk estimation. RadioGraphics 30(2):13-22 nancy on offspring outcomes. Prev Med 50:13-18

2. Amato F, Lopez A, Pena-Mendez AM, Vanhara P, Hampl A, Havel 
5. Barwad A, Dey P, Susheilia S (2012) Artificial neural network in diagnosis of metastatic carcinoma in effusion cytology. Cytom B Clyn Cytom 82:107-111

6. Basheer IA, Hajmeer M (2000) Artificial neural networks: fundamentals, computing, design, and Application. J Microbiol Methods 43:3-31

7. Basso O, Olsen J, Christensen K (1999) Study of environmental, social, and paternal factors in preterm delivery using sibs and half sibs. A population-based study in Denmark. J Epidemiol Commun Health 53:20-23

8. Baxt WG (1995) Application of artificial neural networks to clinical medicine. Lancet 346:1135-1138

9. Berkowitz GS (1981) An epidemiologic study of preterm delivery. Am J Epidemiol 113:81-92

10. Bernstein IM, Mongeon J, Badger G, Solomon L, Heil SH, Higgings ST (2005) Maternal smoking and its association with birth weight. Obstet Gynecol 106:986-991

11. Cazzaniga M, Salerno F, Borroni G, Ceriani R, Stucchi G, Guerzoni $P$ et al (2019) Prediction of asymptomatic cirrhosis in chronic hepatitis C patients: accuracy of artificial neural networks compared with logistic regression models. Eur J Gastroenterol Hepatol 21:681-687

12. Chavi S, Vesna GS, Bush J (2000) Effect of maternal weight gain on infant birth weight. J Perinat Med 28:428-431

13. Cook GD, Strachan DP (1999) Health effects of passive smoking-10. Summary of effects of parental smoking on the respiratory health of children and implications for research. Thorax 54:357-566

14. Cucchetti A, Piscaglia F, Grigioni AD, Ravaioli M, Cescon M, Zanello M et al (2011) Preoperative prediction of hepatocellular carcinoma tumour grade and micro-vascular invasion by means of artificial neural network: a pilot study. J Hepatol 52:880-888

15. Devi MA, Ravi S, Vaishnavi J, Punitha S (2016) Classification of cervical cancer using artificial neural networks. Proc Comput Sci 89:465-472

16. Dey P, Lamba A, Kumari S, Marwaha N (2012) Application of an artificial neural network in the prognosis of chronic myeloid leukemia. Anal Quant Cytol Histol 33:335-339

17. de Sanjose S, Roman E (1991) Low birth weight, preterm, and small for gestational age babies in Scotland, 1981-1984. J Epidemiol Commun Health 45(3):207-210

18. Er O, Temurtas F, Tanrkulu A (2008) Tuberculosis disease diagnosis using artificial neural networks. J Med Syst 34:299-302

19. Hofhuis W, de Jongste JC, Merkus PJ (2003) Adverse health effects of prenatal and postnatal tobacco smoke exposure on children. Arch Dis Child 88:1086-1090

20. Hon-Yi S, Jinn-Tsong T, Wen-Hsien H, Shih-Chin W, I-Te C, KingTeh L (2012) Comparison of artificial neural network and logistic regression models for predicting in-hospital survival after hepatocellular carcinoma surgery. In: SICE annual conference, 20-23 Aug 2012, Akita University, Akita, Japan

21. Hopfield JJ (1982) Neural networks and physical systems with emergent collective computational abilities. Proc Nat Acad Sci 79:2554-2558

22. Hosmer DW, Taber S, Lemeshow S (1981) The importance of assessing the fit of logistic regression models: a case study. Am J Public Health 81:1630-1635

23. Iraji MS (2017) Prediction of post-operative survival expectancy in thoracic lung cancer surgery with soft computing. J Appl Biomed 15:151-159

24. Itchhaporiaa D, Snow PB, Almassy RJ, Oetgena WJ (1996) Artificial neural networks:current status in cardiovascular medicine. J Am Coll Cardiol 28:515-521

25. Kanbayashi Y, Ishikawa T, Kanazawa M, Nakaijima Y, Kawano R, Tabuchi $Y$ et al (2018) Predictive factors in patients eligible for pegfilgrastim prophylaxis focusing on RDI using ordered logistic regression analysis. Med Oncol 35:55. https://doi.org/10.1007/ s12032-018-1116-5

26. Khan J, Wei JS, Ringer M, Saal LH, Ladanyi M, Westermann F et al (2001) Classification and diagnostic prediction of cancers using gene expression profiling and artificial neural networks. Nat Med 7:673-679

27. Kohonen T (1982) Self-organised formation of topologically correct feature maps. Biol Cybern 43:59-69

28. Kosko B (1992) Neural networks and fuzzy systems. PrenticeHall, Englewood Cliffs

29. Landau LI (2001) Parental smoking: asthma and wheezing illnesses in infants and children. Paediatr Respir Rev 2:202-206

30. Lisbao PJ, Taktak AF (2006) The use of artificial neural networks in decision support in cancer: a systematic review. Neural Netw 19(4):408-415

31. McCulloch WS, Pitts W (1943) A logical calculus of the ideas immanent in nervous activity. Bull Math Biophys 5:115-33

32. Papik K, Molnar B, Schaefer R, Dombovari Z, Tulassay Z, Feher $J$ (1998) Application of neural networks in medicine: a review. Med Sci Monit 4:538-546

33. Parker JD, Schoendorf KC, Kiely JL (1994) Associations between measures of socioeconomic status and low birth weight, small for gestational age, and premature delivery in the United States. Ann Epidemiol 4:271-278

34. Peacock JL, Bland JM, Anderson HR (1995) Preterm delivery: effects of socioeconomic factors, psychological stress, smoking, alcohol, and caffeine. Br Med J 311:531-535

35. Ramesh AN, Kambhampati C, Monson JRT, Drew PJ (2004) Artificial intelligence in medicine. Ann R Coll Surg Engl 86:334-338. https://doi.org/10.1308/147870804290

36. Solis-Paredes M, Estrada-Gutierrez G, Perichart-Perera O, Guzman-Huerta M, Borboa-Olivares $\mathrm{H}$, Bravo-Flores E et al (2018) Key clinical factors predicting adipokine and oxidative stress marker concentrations among normal, overweight and obese pregnant women using artificial neural networks. Int J Mol Sci 19(1):86. https://doi.org/10.3390/ijms19010086

37. Temurtas F (2009) A comparative study on thyroid disease diagnosis using neural networks. Expert Syst Appl 36:944-949

38. Terziolu F, Yücel Ç (2008) Sigarann gebelik ve bebek salna etkileri. Salcakla Dergisi 5:10-15 (in Turkish)

39. Thompson JM, Irgens LM, Rasmussen S, Daltveit AK (2006) Secular trends in socio-economic status and the implications for preterm birth. Pediatr Perinat Epidemiol 20(3):182-187

40. Torres A, Nieto JJ (2006) Fuzzy logic in medicine and bioinformatics. J Biomed Biotechnol: 1-7, Article ID 91908

41. Wang C, Li L, Wang L, Ping Z, Flory MT, Wang G et al (2013) Evaluating the risk of type 2 diabetes mellitus using artificial neural network: an effective classification approach. Diabetes Res Clin Pract 100:111-118

42. Wiest MM, Lee KJ, Carlin JB (2015) Statistics for clinicians: an introduction to logistic regression. J Paediatr Child Health 51:670-673

43. World Health Organization (2010) International statistical classification of diseases and related health problems. WHO, Geneva

44. Global Database on Body Mass Index (2018) World Health Organization Web site. http://apps.who.int/bmi/index.jsp?intro Page=intro_3.html. Published 2018. Accessed 30 Nov 2018

45. The American College of Obstetricians and Gynecologists (ACOG) (1993) Nutrition during pregnancy. The American College of Obstetricians and Gynecologists, Washington, Technical Bulletin No. 179

Publisher's Note Springer Nature remains neutral with regard to jurisdictional claims in published maps and institutional affiliations. 